\title{
Avaliação química de substitutos de cacau e estudo sensorial de achocolatados formulados
}

\author{
Chemical evaluation of cocoa substitutes and study of formulated chocolate drink powder
}

\author{
Magda Leite MEDEIROS ${ }^{1}$, Suzana Caetano da Silva LANNES ${ }^{1 \star}$
}

\begin{abstract}
Resumo
O cacau em pó é obtido a partir da pasta de cacau preparada com sementes que passaram pelos processos de fermentação, secagem, torrefação, moagem e prensa (para separação da manteiga de cacau), podendo ser utilizado em formulações de achocolatados que além de cacau em pó são produzidos com: açúcar, aroma e outros ingredientes constantes da formulação. Nos países líderes de produção, o preço do cacau é afetado pelo clima, pragas e fatores políticos. Substitutos de cacau são substâncias que podem entrar na formulação de produtos que utilizam cacau em pó para substituí-lo parcial ou totalmente, com a finalidade de diminuição do preço do produto final, garantia de qualidade dos produtos dependentes de cacau na entressafra ou mesmo na possível ausência do cacau em pó no mercado. Os substitutos escolhidos para avaliação foram: farinha tostada de alfarroba, cupuaçu em pó, composto da semente de cacau e aromas. Os objetivos foram: avaliar quimicamente (composição centesimal e teor de metilxantinas) os substitutos de cacau e sensorialmente, sob a forma de achocolatados. Concluiu-se que os produtos analisados apresentam diferentes propriedades químicas daquelas do cacau, sendo que os aromas foram preferidos nos testes sensoriais.
\end{abstract}

Palavras-chave: cacau; substitutos de cacau; achocolatado.

\begin{abstract}
Cocoa powder is obtained from cocoa paste. It is prepared with fermented, dried, toasted, grinded and pressed beans (to separate cocoa butter). It can be used to produce chocolate powders, which contains cocoa powder, sugar, chocolate flavor and other ingredients. In the major producing countries, the price of cocoa by-product is affected by the climate, plagues and political factors. Cocoa substitutes are substances that can total or partially substitute cocoa powder in the formulation of different products with the purpose of reducing the final price, guaranteeing the quality in the off season or even during a possible short availability of cocoa powder in the market. The substitutes chosen for evaluation include toasted carob powder, cupuaçu powder, cocoa seed compounds and aroma. The objectives of this study were evaluate chemical parameters (centesimal composition and methylxanthines) of the cocoa powder substitutes and carry out a sensorial evaluation of chocolate drink powders formulated with the substitutes. As results, the substitutes showed different chemical properties from those of cocoa, and the sensorial tests showed preference to the aroma.

Keywords: cocoa; cocoa substitute; chocolate powder.
\end{abstract}

\section{Introdução}

O homem tem habilidade natural para comparar, diferenciar e quantificar os atributos sensoriais e a análise sensorial utiliza-se dessa habilidade para avaliar alimentos e bebidas, empregando a metodologia apropriada aos objetivos de estudo e tratamentos estatísticos dos dados. A compreensão dos objetivos sensoriais de cada análise realizada é essencial para a utilização dos testes metodológicos, desenho experimental e tratamentos estatísticos mais apropriados. Basicamente, os métodos sensoriais estão agrupados em analíticos e afetivos (FERREIRA et al., 2000):

- Afetivos: testes de preferência/aceitação (comparação pareada - preferência, ordenação, escala hedônica e escala do ideal);

- Analíticos: testes de diferença ou discriminativos (comparação pareada, triangular, duo-trio, ordenação, comparação múltipla ou diferença do controle) e testes descritivos (perfil de sabor, perfil de textura e análise descritiva quantitativa).
Achocolatados são produtos em pó formulados à base de cacau, açúcar, aroma e outros ingredientes, destinados principalmente para o consumo na forma de bebida acrescido de leite (MEDEIROS, 2006).

Além das metilxantinas, principalmente a teobromina, os produtos derivados de cacau contêm quantidades substanciais de nutrientes como lípides, carboidratos e proteínas (BELITZ; GROSCH, 1999). Hurst et al. (2002) comentaram que o cacau possui composição química única, com mais de 500 compostos, dentre os quais merecem destaque as metilxantinas. Classificadas como alcaloides purínicos, são consideradas substâncias estimulantes, e as encontradas no cacau são: teobromina, em maior concentração, seguida da cafeína e por último da teofilina.

Matissek (1997) apontou as razões de interesse no grupo das metilxantinas: contribuem com o sabor amargo do chocolate, juntamente com compostos formados durante a torrefação; servem

Recebido para publicação em 11/5/2007

Aceito para publicação em 14/2/2009 (002520)

1 Departamento de Tecnologia Bioquímico-Farmacêutica, Faculdade de Ciências Farmacêuticas, Universidade de São Paulo - USP, Av. Prof. Lineu Prestes, 580, Bloco 16, Cidade Universitária, CEP 05508-900, São Paulo-SP, Brasil, E-mail: scslan@usp.br

${ }^{*}$ A quem a correspondência deve ser enviada 
como parâmetros de qualidade, pois atestam a presença de cacau (o cacau é a única planta americana que possui teobromina como alcaloide principal). Ainda, possuem efeitos farmacológicos sobre os sistemas: nervoso, cardiovascular, gastrintestinal, respiratório e renal (CAUDLE; BELL, 2000).

Devido ao alto teor de gordura (10-25\%), à elevada quantidade de polissacarídeos hidrofóbicos e a sua estrutura capilar, que aprisiona bolhas de ar, o cacau é um ingrediente de baixa solubilidade (OMOBUWAJO; BUSARI; OSEMWEGIE, 2000).

Os achocolatados geralmente são formulados com cerca de 70\% de açúcar, principalmente sacarose, e 30\% de cacau em pó. De acordo com Omobuwajo, Busari e Osemwegie (2000), outros ingredientes comuns em formulações de bebidas achocolatadas comerciais são: extrato de malte, leite em pó, ovos, vitaminas e minerais.

Existem no mercado diversas formulações de achocolatado (como os: diet, light e saborizados), sendo a maioria destinada a crianças e adolescentes. Contudo a aceitação dos achocolatados não se restringe somente a esse público. Logo, os achocolatados podem ser usados como veículo para a complementação alimentar de pessoas com carências nutricionais (MEDEIROS, 2006).

Quanto ao seu processamento, Eduardo (2005) enumerou as seguintes possibilidades:

- Mistura simples: os ingredientes, de tamanhos padronizados, são misturados até a uniformidade;

- Spray-drying: processo de secagem através de pulverização, no qual gotículas da solução entram em contato com um fluxo de ar quente, permitindo a rápida evaporação; garante a uniformização do tamanho das partículas e contribui para a instantaneização dos achocolatados (LANNES; MEDEIROS, 2003) e;

- Aglomeração: processo de secagem realizado em leito fluidizado que contribui, como o processo anterior, com a instantaneização, além de melhorar a aparência, o fluxo e a homogeneidade dos produtos finais.

Os substitutos de cacau são substâncias que podem entrar na formulação de biscoitos, massas para panificação, sobremesas, sorvetes, bebidas lácteas e achocolatados, para substituir parcial ou totalmente o cacau, com o intuito de garantir a qualidade do produto final durante a entressafra, diminuir o preço ou acrescentar alguma nova característica ao produto final. Dentre os possíveis substitutos, destacam-se:

- Farinha tostada de alfarroba: fruto da alfarrobeira (Ceratonia siliqua L.), árvore nativa dos países mediterrâneos, a alfarroba é consumida em muitos países árabes sob a forma de doces e de bebida durante o Ramadan (YOUSIF; ALGHZAWI, 2000). A polpa após secagem, trituração e torrefação dá origem ao pó ou farinha de alfarroba, que possui cor e flavour similares ao cacau. Os compostos estimulantes (cafeína e teobromina) que são encontrados no cacau e considerados por diversos autores como fatores antinutricionais responsáveis por efeitos fisiológicos adversos (GROSSO; BRACKEN, 2005; BONVEHÍ; COLL, 2000; CHEN et al., 1998; ETENG et al.,1997), não estão presentes na farinha de alfarroba, além disso, possui baixo custo quando comparada ao cacau e graças a sua composição (rica em carboidratos de baixo peso molecular) apresenta boa solubilidade (OWEN et al., 2003;
YOUSIF; ALGHZAWI, 2000; BRAND, 1984). Apesar de tais vantagens, segundo Kumazawa et al. (2002), a maior parte da alfarroba ainda é descartada.

- Cupuaçu em pó: o cupuaçu Theobroma grandiflorum Schum, pertence ao mesmo gênero, Theobroma, do cacau, são dois frutos filogeneticamente próximos, mas morfologicamente distintos. O parentesco entre eles pode ser confirmado através da composição e das propriedades bioquímicas de suas sementes, além da presença dos alcaloides purínicos (metilxantinas) nelas encontrados. Os híbridos das duas espécies são capazes de produzir grande quantidade de frutos (REISDORFF et al., 2004; YANG et al., 2003). A polpa do cupuaçu corresponde de 35 a $40 \%$ da fruta e é a maior responsável pelo seu custo (YANG et al., 2003, NAZARÉ, 2000; QUEIROZ; GARCIA, 1999). Já as sementes do cupuaçu constituem cerca de $20 \%$ da massa total da fruta e, quando beneficiadas, da mesma forma que as de cacau, desenvolvem aroma semelhante ao de chocolate.

- Aromas em pó: substâncias que conferem sabor e/ou odor, potencializam ou padronizam o sabor inicial dos alimentos, repõem perdas de processo, mascaram sabores indesejáveis, criam sensações novas e otimizam os custos dos alimentos. Os aromas são conjuntos de princípios ativos como ésteres, ácidos, cetonas, aldeídos, alcoóis e terpenos, usados em quantidades mínimas associados a um veículo (DUTCOSKY, 2006). Em formulações alimentícias, o cacau é responsável pela cor, aroma, sabor e volume do produto final (GRAB; BRUGGER; TAYLOR, 1978). Para que um aroma possa atuar como substituto de cacau ele deve conferir, além dos atributos sensoriais, volume para o produto final.

O objetivo foi caracterizar quimicamente (composição centesimal, $\mathrm{pH}$ e teor de metilxantinas: teobromina, cafeína e teofilina) produtos comercialmente utilizados como substitutos de cacau e verificar sua aceitação (através de análise sensorial dos tipos afetiva quantitativa e discriminativa) sob a forma de achocolatados.

\section{Material e métodos}

\subsection{Material}

Foram utilizados substitutos de cacau obtidos do mercado, além de cacau em pó, das seguintes empresas:

- Cacau em pó lecitinado IMPACT LEC - AW 70: Cargill Agrícola S.A.;

- Aroma de Chocolate RBP 10886: Saporitti do Brasil Ltda.;

- Aroma Idêntico ao Natural de Cacau Cocoa Extender: IFF Essências e Fragrâncias Ltda.;

- Composto de Cacau (Fibracau Natural e Fibracau Preta): Cacau \& Cia;

- Cupuaçu em pó: Projeto RECA - Reflorestamento Econômico Consorciado Adensado - Amazônia;

- Farinha Tostada de Alfarroba (Carovit D e Carovit ED): Great Foods Brasil. 


\subsection{Métodos}

Avaliação química

Umidade

A umidade das amostras foi determinada por gravimetria, de acordo com metodologia AOAC (1990), para análise de sementes de cacau e seus produtos, utilizando-se estufa a $105,0{ }^{\circ} \mathrm{C}$ e dessecador sob vácuo para resfriamento. A secagem foi efetuada até massa constante.

Teor de lípides

A quantificação foi realizada pelo método de extração de Soxhlet, segundo metodologia AOAC (1990).

\section{Nitrogênio total e proteínas}

A quantificação do teor de nitrogênio foi feita através de metodologia Micro-Kjeldahl AOAC (1990) modificada, sendo que a porcentagem proteica das amostras foi encontrada multiplicando-se a porcentagem de nitrogênio pelo fator de conversão 6,25.

\section{Cinzas}

A determinação de cinzas foi realizada de acordo com metodologia do Instituto Adolfo Lutz (1976).

$\mathrm{pH}$

$\mathrm{O}$ pH das amostras foi determinado a partir de soluções a $10 \%(\mathrm{p} / \mathrm{p})$ das amostras e água destilada. Para tanto, utilizou-se medidor de pH-Analyser Modelo 300M (Analyser Comércio e Indústria Ltda.), empregando-se eletrodo tipo penetração modelo 2A04-IF (Analyser Digimed).

Teor de metilxantinas

A extração das metilxantinas foi realizada através de maceração sucessiva em etanol, utilizaram-se para tanto 10,00 g de amostra. Os extratos obtidos eram concentrados sucessivamente (em evaporador rotatório a vácuo) e o álcool reaproveitado, até verificação da ausência de alcaloides no macerado por reação negativa com reagente de Dragendorff. A partir do extrato concentrado resultante foram extraídas as metilxantinas. $\mathrm{O}$ método empregado leva em consideração a natureza básica dos alcaloides. Consiste, num primeiro passo, na partição do extrato bruto entre uma fase orgânica e uma fase aquosa ácida; a fase orgânica é então descartada e a aquosa é alcalinizada. Realiza-se uma nova partição através da adição de uma nova fase orgânica (clorofórmio). A fase orgânica obtida foi seca em evaporador rotatório a vácuo e o sólido obtido é uma mistura dos alcaloides presentes na amostra inicial (VERPOORTE, 1986).

\section{Avaliação sensorial}

A avaliação sensorial das formulações foi realizada em duas etapas. A primeira, um teste afetivo, serviu para selecionar a melhor formulação e a segunda etapa, composta por um teste discriminativo, serviu para confrontar as formulações com e sem substituição de cacau. O projeto foi aprovado pelo Comitê de Ética em Pesquisas da Faculdade de Ciências Farmacêuticas da USP.

Teste afetivo quantitativo

Foi realizado um teste afetivo quantitativo com escala hedônica estruturada de 7 pontos ( 1 = desgostei muitíssimo até 7 = gostei muitíssimo) com 72 provadores não treinados, que se declararam consumidores de achocolatado. Antes da degustação os provadores foram instruídos a ler e assinar o cadastro de consumidores, declarando-se não alérgicos aos componentes das formulações, permitindo o uso da informação prestada para seu devido fim e também possuidores do direito de desistir de participar a qualquer momento do teste.

A avaliação, realizada em cabines individuais no Laboratório de Análise Sensorial da Faculdade de Ciências Farmacêuticas/USP, foi planejada de forma que cada um dos participantes provasse as 4 amostras servidas sequencialmente em blocos completos balanceados, com relação à ordem de apresentação.

Os achocolatados foram preparados com leite integral e mantidos em geladeira até o momento de servir, na diluição de 100,0 g. $\mathrm{L}^{-1}$. Pediu-se aos provadores que entre uma amostra e a seguinte provassem água e biscoito de sabor neutro (água e sal) para diminuir a interferência entre os achocolatados. Após provar cada amostra os participantes deveriam preencher a ficha de avaliação que era fornecida junto com a primeira amostra.

\section{Teste discriminativo triangular}

A segunda etapa da análise foi realizada entre a amostra que obteve a maior nota e a formulação que não continha substituição de cacau.

Testes discriminativos são usados para determinar se existe diferença perceptível entre amostras. O teste escolhido foi o triangular, no qual o provador deveria escolher qual dentre 3 amostras era a diferente. A vantagem do teste triangular é a menor possibilidade de acerto ao acaso Ferreira et al. (2000).

Os 60 provadores selecionados passaram pelo mesmo procedimento descrito anteriormente.

As amostras numeradas foram apresentadas ao provador juntas, o qual foi instruído a prová-las da esquerda para a direita e marcar na ficha de avaliação fornecida a amostra diferente.

\section{Formulação dos achocolatados}

As formulações (ingredientes e quantidades) dos achocolatados encontram-se na Tabela 1. As quantidades de substitutos utilizadas nas formulações com os Aromas Saporitti e Cocoa Extender foram as recomendadas pelos fornecedores; para outras amostras foram realizados testes preliminares para a escolha da porcentagem de cacau substituída. Depois de pesados, os ingredientes permaneceram sob agitação em misturador tipo tambor durante 15 minutos, tempo necessário para a homogeneização dos pós. Os ingredientes foram misturados com 2 dias de antecedência.

As formulações contendo os substitutos foram calculadas tendo como base a formulação inicial, que continha somente 
cacau. Nessa formulação, mesmo sendo constituída de 70\% de açúcar, verificou-se que o sabor doce não era compatível com as formulações comerciais; para corrigir a falta de dulçor foi acrescentado aspartame em todas as amostras.

\section{Resultados e discussão}

\subsection{Análise da composição centesimal}

A determinação da composição centesimal é uma ferramenta útil para a caracterização química dos substitutos e comparação deles com o cacau.

O coeficiente de variação (CV), ou desvio padrão relativo, é uma maneira de se verificar estatisticamente a precisão de um método. O CV quantifica a dispersão dos valores experimentais, ele é definido através da Equação 1:

$C V=\frac{d p}{m} \times 100 \%$

em que:

$$
\begin{aligned}
& \mathrm{dp}=\text { desvio padrão, } \\
& \mathrm{m}=\text { média. }
\end{aligned}
$$

Os resultados da composição centesimal e seus respectivos coeficientes de variação estão representados graficamente na Figura 1, e listados na Tabela 2.

As exceções são as amostras AS e CE, os aromas, para a determinação do extrato etéreo total. Mesmo depois de 5 repetições

Tabela 1. Formulações dos achocolatados.

\begin{tabular}{lcrrrr}
\hline Ingrediente (\%) & Cacau & AS & CE & CD & CP \\
\hline Açúcar & 70,0 & 70,0 & 70,0 & 60,0 & 62,7 \\
Cacau & 27,7 & 20,0 & 20,0 & 24,0 & 24,0 \\
Matoldextrina $^{1}$ & 0,0 & 2,7 & 1,2 & 3,2 & 0,0 \\
Substituto $^{2}$ & 0,0 & 4,0 & 6,5 & 10,0 & 10,0 \\
Aroma $^{2}$ & 2,0 & 3,0 & 2,0 & 12,5 & 3,0 \\
Sal & 0,2 & 0,2 & 0,2 & 0,2 & 0,2 \\
Aspartame & 0,1 & 0,1 & 0,1 & 0,1 & 0,1 \\
\hline
\end{tabular}

AS = Aroma de Chocolate RBP 10886 (Aroma Saporitti); CE = Aroma Idêntico ao Natural de Cacau (Cocoa Extender); $\mathrm{CD}$ = Farinha Tostada de Alfarroba; $\mathrm{CP}=$ Cupuaçu em pó;

${ }^{1}$ Utilizada para completar as formulações em $100 \%$; e ${ }^{2}$ Aroma Duas Rodas. desse ensaio para cada amostra, os CVs encontrados foram 47,14 e 23,57, respectivamente. Pode-se concluir que a metodologia escolhida para a determinação do extrato etéreo não foi adequada para essas duas amostras.

As amostras AS e CE apresentaram composição centesimal estatisticamente igual; possivelmente os componentes utilizados para "dar corpo" às amostras sejam os mesmos. Nos laudos técnicos que acompanhavam as amostras não estavam descriminados seus ingredientes, logo não se pôde saber qual seria esse composto. A única diferença foi o teor

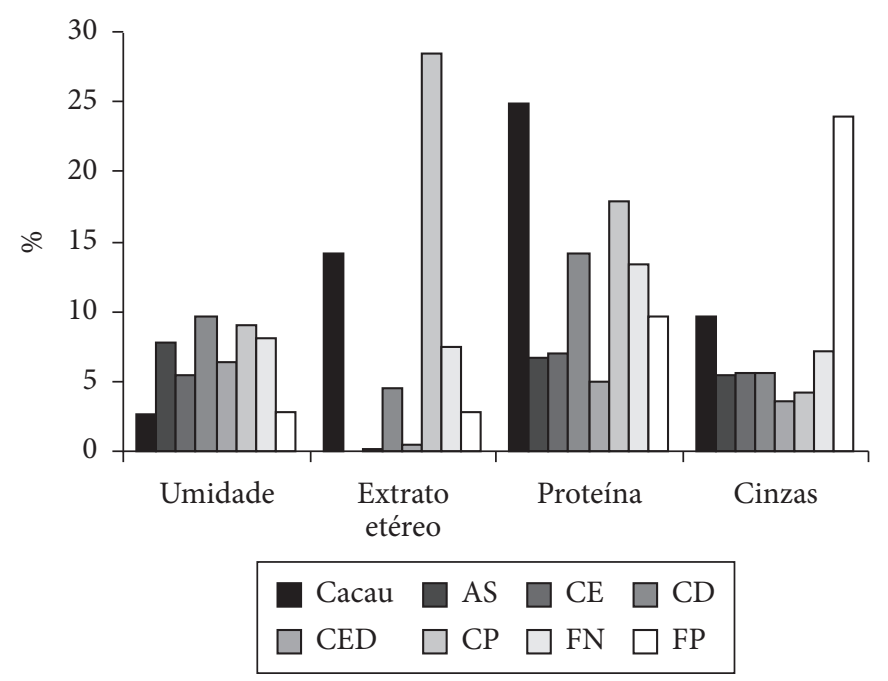

Figura 1. Representação gráfica dos valores da composição centesimal das amostras. Os coeficientes de variação encontram-se, na sua maioria, abaixo de 5 , significando que os resultados obtidos durante os ensaios não variaram mais de que $5 \%$ entre si. As exceções são as amostras AS e CE, os aromas, para a determinação do extrato etéreo total. Mesmo depois de 5 repetições desse ensaio para cada amostra, os CVs encontrados foram 47,14 e 23,57, respectivamente. Pode-se concluir que a metodologia escolhida para a determinação do extrato etéreo não foi adequada para essas duas amostras. AS = Aroma de Chocolate RBP 10886 (Aroma Saporitti); CE = Aroma Idêntico ao Natural de Cacau (Cocoa Extender); FN = Composto de Cacau (Fibracau Natural); FP = Composto de Cacau (Fibracau Preta); CD = Farinha Tostada de Alfarroba (Carovit D); CED = Farinha Tostada de Alfarroba (Carovit ED); e CP = Cupuaçu em pó.

\begin{tabular}{|c|c|c|c|c|c|c|c|c|c|}
\hline & Umidade $\pm \delta(\%)$ & $\mathrm{CV}$ & Extrato etéreo $\pm \delta(\%)$ & $\mathrm{CV}$ & Proteína $\pm \delta(\%)$ & $\mathrm{CV}$ & Cinzas $\pm \delta(\%)$ & $\mathrm{CV}$ & Carboidratos $^{1}(\%)$ \\
\hline Cacau & $2,68 \pm 0,07^{a}$ & 2,64 & $14,21 \pm 0,03^{\mathrm{f}}$ & 0,25 & $24,84 \pm 0,11^{\mathrm{g}}$ & 0,46 & $09,69 \pm 0,12^{e}$ & 1,27 & 48,58 \\
\hline AS & $7,79 \pm 0,34^{c}$ & 4,36 & $00,05 \pm 0,02^{\mathrm{a}}$ & 47,14 & $06,73 \pm 0,23^{b}$ & 3,43 & $05,41 \pm 0,19^{c}$ & 3,53 & 80,02 \\
\hline $\mathrm{CE}$ & $5,51 \pm 0,23^{b}$ & 4,24 & $00,12 \pm 0,03^{\mathrm{a}}$ & 23,57 & $06,92 \pm 0,19^{\mathrm{b}}$ & 6,92 & $05,64 \pm 0,10^{c}$ & 1,76 & 81,81 \\
\hline CED & $6,39 \pm 0,28^{b}$ & 4,43 & $00,40 \pm 0,01^{\mathrm{b}}$ & 1,79 & $04,97 \pm 0,05^{\mathrm{a}}$ & 1,06 & $03,54 \pm 0,06^{\mathrm{a}}$ & 1,80 & 84,70 \\
\hline $\mathrm{CP}$ & $9,06 \pm 0,06^{d}$ & 0,70 & $28,38 \pm 1,07^{g}$ & 3,76 & $17,88 \pm 0,25^{\mathrm{f}}$ & 1,41 & $04,23 \pm 0,00^{\mathrm{b}}$ & 0,00 & 40,45 \\
\hline
\end{tabular}

Tabela 2. Resultados da composição centesimal.

$\mathrm{n}=3 ; \delta=$ desvio padrão; AS = Aroma de Chocolate RBP 10886 (Aroma Saporitti); CE = Aroma Idêntico ao Natural de Cacau (Cocoa Extender); FN = Composto de Cacau (Fibracau Natural); FP = Composto de Cacau (Fibracau Preta); CD = Farinha Tostada de Alfarroba (Carovit D); CED = Farinha Tostada de Alfarroba (Carovit ED); CP = Cupuaçu em pó; ${ }^{1}$ calculado por diferença; e Índices diferentes, na mesma coluna, indicam diferença significativa ao nível de $5 \%$. 
de umidade. Esta diferença pode ser explicada pela maior higroscopicidade, observada durante os ensaios, apresentada pela amostra AS.

Por se tratar de dois aromas compostos por princípios ativos (agentes aromatizantes e saborizantes) e um veículo, o teor de proteína determinado está bem elevado. Como o mesmo foi calculado através da determinação do nitrogênio total (método Kjeldahl), esse nitrogênio pode ser oriundo do veículo ou mesmo de algum outro princípio ativo utilizado. $\mathrm{O}$ fornecedor da amostra AS, em comunicação pessoal, afirmou que ela contém em sua formulação casca de semente de cacau em pó, portanto parte do teor de nitrogênio pode ser atribuída também à presença de teobromina.

As farinhas de alfarroba utilizadas para as análises apresentaram diferença em relação à coloração. A amostra CED provavelmente passou por um processo de torrefação mais severo, pois possui coloração mais escura.

Houve diferença significativa (5\%) entre as amostras de farinha de alfarroba (CD e CED), para todos os itens analisados. Como esperado (YOUSIF; ALGHZAWI, 2000; BRAND, 1984), os valores da composição centesimal das farinhas, exceto a umidade, apresentaram-se inferiores em relação aos do cacau.

É exatamente o menor teor de lípides, associado à ausência de outros dos compostos antinutricionais (as metilxantinas), que é apontado como a vantagem da utilização da alfarroba em relação ao cacau em pó.

Yousif, Alghzawi (2000) determinaram em seu trabalho a composição centesimal da farinha de alfarroba tostada. Os resultados comparados com os encontrados para as amostras CD e CED estão graficamente representados na Figura 2.

Comparando-se a composição da amostra CD com dados encontrados na literatura para farinha de alfarroba não tostada, nota-se que é bem distinta (cerca de 15 vezes) em relação ao extrato etéreo $(\mathrm{CD}=4,5 \%$ e YOUSIF e ALGHZAWI $=0,3 \%)$, e ao teor de proteína, cerca de 3 vezes ( $C D=14,1 \%$ e YOUSIF

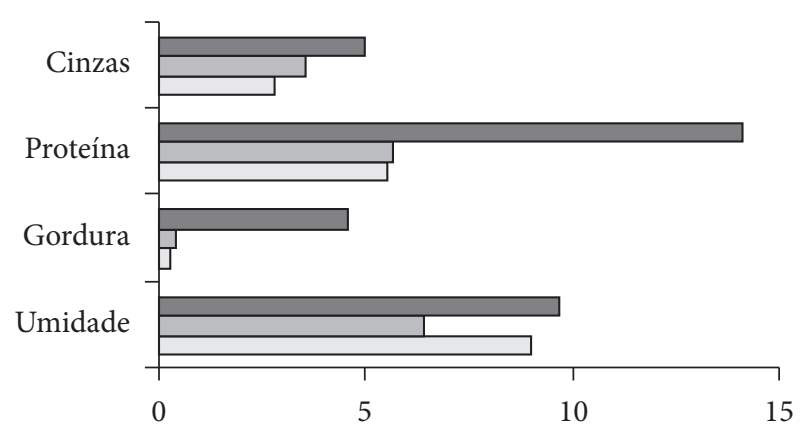

(\%)

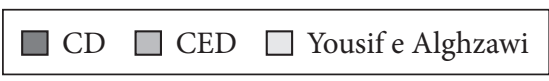

Figura 2. Comparação gráfica da composição centesimal das farinhas de alfarroba analisadas com a da literatura; $C D=$ Farinha Tostada de Alfarroba (Carovit D); e CED = Farinha Tostada de Alfarroba (Carovit ED). e ALGHZAWI $=5,5 \%)$. Como os CVs dessa amostra, para as análises realizadas, foram inferiores a 5 , pode-se concluir que ela possui conteúdo de açúcares e fibras inferior, tanto em relação aos dados da literatura, como quanto à amostra CED.

Os resultados encontrados para o pó de cupuaçu estão de acordo com os apresentados por MEDEIROS et al. (2006). O teor de lípides do pó é o dobro do apresentado pelo cacau. Isso prejudica a molhabilidade, além de aumentar o valor calórico de seus produtos.

Assim como ocorreu com as farinhas de alfarroba, os compostos de cacau (que possuem casca em sua composição) diferiram entre si em todos os itens analisados.

Também como nas alfarrobas, a diferença das amostras de compostos está no grau de torrefação. O excesso de torrefação, durante o processamento, da amostra FP pode explicar o alto teor de cinzas encontrado $(24,0 \%)$ da seguinte forma: a perda de parte da matéria orgânica, sob a forma de $\mathrm{CO}$ e $\mathrm{CO}_{2}$, pode aumentar a razão minerais/compostos orgânicos.

O cacau foi a amostra analisada com maior teor proteico, seguida do pó de cupuaçu, farinha de alfarroba $\mathrm{CD}$, composto de cacau FN, composto de cacau FP, aroma CE, aroma AS e farinha de alfarroba CED.

Todavia, a análise escolhida para a quantificação do teor de proteína (Kjeldahl) determina o nitrogênio total, então parte desse nitrogênio quantificado tem origem não proteica, de compostos como as metilxantinas. Logo, os valores de proteína podem estar superestimados, principalmente para as amostras FP e FN.

\section{$3.2 \mathrm{pH}$}

A Tabela 3 apresenta os valores de $\mathrm{pH}$ das amostras e da água destilada utilizada no seu preparo.

$\mathrm{O}$ valor de $\mathrm{pH}$ do cacau apresentou-se muito próximo à neutralidade, confirmando assim que a amostra de cacau utilizada fora previamente alcalinizada. As outras amostras apresentaram-se ácidas (pH entre 3,56 e 5,74), com exceção da amostra FP.

Tabela 3. Valores de $\mathrm{pH}$ das amostras.

\begin{tabular}{lc}
\hline & $\mathrm{pH}$ \\
\hline Cacau & 7,04 \\
$\mathrm{AS}$ & 4,74 \\
$\mathrm{CE}$ & 3,56 \\
$\mathrm{CD}$ & 5,70 \\
$\mathrm{CED}$ & 4,50 \\
$\mathrm{CP}$ & 5,74 \\
$\mathrm{FN}$ & 4,90 \\
$\mathrm{FP}$ & 8,66 \\
Água destilada & 7,04 \\
\hline $\mathrm{n}=2$; AS $=$ Aroma de Chocolate RBP 10886 (Aroma Saporitti); CE = Aroma Idêntico \\
ao Natural de Cacau (Cocoa Extender); FN = Composto de Cacau (Fibracau Natural); \\
FP = Composto de Cacau (Fibracau Preta); CD = Farinha Tostada de Alfarroba (Carovit D); \\
CED = Farinha Tostada de Alfarroba (Carovit ED); e CP = Cupuaçu em pó.
\end{tabular}


Os valores apresentados por Yousif, Alghzawi (2000) para a farinha de alfarroba não tostada e tostada (5,96 e 4,81, respectivamente) são bem semelhantes aos encontrados para as farinhas testadas.

\subsection{Teor de metilxantinas}

Metilxantinas são alcaloides purínicos que possuem atividade biológica. Os representantes mais conhecidos dessa classe de compostos são: cafeína, teobromina e teofilina. Esses compostos também são os principais alcaloides presentes no cacau e derivados (THOMAS et al., 2004).

O extrato total obtido de cada amostra foi determinado gravimetricamente, após extração e secagem dos solventes, e estão listados na Tabela 4.

Como esperado, o teor de alcaloides do cacau foi superior ao encontrado nos substitutos. Matissek (1997) apresentou os seguintes valores de teobromina e cafeína 1,42 e 0,07\%, respectivamente.

O teor de metilxantinas da amostra de cupuaçu em pó representa apenas $42,20 \%$ do encontrado para o cacau,

Tabela 4. Extrato total.

\begin{tabular}{lc}
\hline & $\%$ \\
\hline Cacau & 1,09 \\
AS & 0,05 \\
CE & 0,28 \\
CD & 0,41 \\
CED & 0,24 \\
CP & 0,46 \\
FN & 0,54 \\
FP & 0,32 \\
\hline
\end{tabular}

$\mathrm{n}=1$; AS: Aroma de Chocolate RBP 10886 (Aroma Saporitti); CE = Aroma Idêntico ao Natural de Cacau (Cocoa Extender); FN = Composto de Cacau (Fibracau Natural); FP = Composto de Cacau (Fibracau Preta); CD = Farinha Tostada de Alfarroba (Carovit D); $\mathrm{CED}=$ Farinha Tostada de Alfarroba (Carovit ED); e CP = Cupuaçu em pó.

Tabela 5. Notas do teste de ordenação da preferência.

\begin{tabular}{lccc}
\hline & Somatória & Média & Variância \\
\hline $\mathrm{CD}$ & 295 & 4,10 & 2,29 \\
$\mathrm{CP}$ & 262 & 3,64 & 2,01 \\
$\mathrm{As}$ & 311 & 4,32 & 2,00 \\
$\mathrm{CE}$ & 345 & 4,79 & 1,86 \\
\hline
\end{tabular}

Número de provadores: 72; AS = Aroma de Chocolate RBP 10886 (Aroma Saporitti); $\mathrm{CE}=$ Aroma Idêntico ao Natural de Cacau (Cocoa Extender); $\mathrm{CD}=$ Farinha Tostada de Alfarroba; e $\mathrm{CP}=$ Cupuaçu em pó.

Tabela 6. Resultado do teste de aceitabilidade das amostras de achocolatados.

\begin{tabular}{ccccc}
\hline & CD & CP & AS & CE \\
\hline Média & $4,10^{\text {ab }}$ & $3,64^{\mathrm{a}}$ & $4,32^{\text {bc }}$ & $4,79^{\mathrm{c}}$ \\
\hline AS = Aroma de Chocolate RBP & 10886 (Aroma Saporitti); CE $=$ Aroma Idêntico ao Natural de \\
Cacau (Cocoa Extender); CD = Farinha Tostada de Alfarroba; e CP = Cupuaçu em pó.
\end{tabular}

confirmando resultados de trabalhos anteriores (MEDEIROS; LANNES, 1999; LANNES; MEDEIROS, 2003).

O mesmo pôde ser observado para os outros substitutos, já que todos apresentaram valores inferiores ao cacau, com ênfase para a amostra de aroma AS.

Por se tratar de compostos que possuem atividades farmacológicas (aumento da diurese, estímulo do sistema nervoso central e circulatório, entre outros), a baixa concentração das metilxantinas nos substitutos apresenta mais uma vantagem de utilização dos substitutos quando se deseja diminuir o valor desses compostos em formulações sabor chocolate.

A técnica de extração empregada permite separar, além da teobromina, cafeína e teofilina, compostos que possuem comportamento ácido-base semelhante ao dos alcaloides, como aminas e outros compostos nitrogenados.

Essa é a explicação para os valores encontrados para as amostras de farinha de alfarroba (CD e CED); já que a alfarroba, sabidamente, não possui metilxantinas em sua composição (YOUSIF; ALGHZAWI, 2000; KUMAZAWA et al., 2002).

\subsection{Avaliação sensorial}

A somatória, média e variância das notas obtidas no teste afetivo quantitativo estão na Tabela 5. A partir desses valores foi realizada a análise de variância (ANOVA).

Como o valor de F calculado $(8,12)$ foi maior que o F crítico $(2,64)$, podemos afirmar que houve diferença significativa ao nível de erro de $5 \%$ entre os tratamentos. Para determinar quais amostras eram diferentes entre si foi aplicado o teste Tukey. Os resultados estão listados na Tabela 6 .

Índices diferentes na mesma linha indicam diferença significativa ao nível de $5 \%$.

As amostras de aromas obtiveram as maiores notas, portanto são as mais aceitas.

A amostra CE foi a selecionada para o teste triangular, realizado com a formulação que não continha substituto.

No teste triangular com 60 provadores, o número mínimo de respostas corretas para estabelecer diferença significativa, com 5\% de nível de significância é 27, Ferreira et al. (2000).

Foram obtidas 35 respostas corretas, portanto foi detectada a diferença entre as amostras. Contudo, tal diferença pode ser atribuída à diferença de coloração que as formulações apresentaram após o preparo; já que no espaço reservado aos comentários essa foi a justificativa de alguns provadores.

\section{Conclusões}

Nenhum dos substitutos analisados apresentou composição química semelhante a do cacau, representando uma vantagem em relação ao teor de metilxantinas, pois produtos formulados com esses substitutos não irão possuir os efeitos farmacológicos inerentes ao cacau.

Os melhores substitutos de cacau foram os aromas comerciais, sendo que para amenizar a diferença observada deve-se adaptar a coloração das formulações. 
Agradecimentos

À CAPES.

\section{Referências bibliográficas}

ASSOCIATION OF OFFICIAL ANALITYCAL CHEMISTS - AOAC. Official methods of analysis . 15 ed. Washington, 1990. p. 763-776.

BELITZ, H. D.; GROSCH, W. Food chemistry. Berlin: Springer Verlag, 1999. p. $702-711$.

BRAND, E. Carob. Nutrition and Food Science, v. 91, n. 6, p. 22-23, 1984.

BONVEHÍ, J. S.; COLL, F. V. Evaluation of purine alkaloids and diketopiperazines contents in processed cocoa powder. European Journal of Food Research Technology, v. 210, n. 3, p. 189-195, 2000.

CAUDLE, A. G.; BELL, L. N. Caffeine and theobromine contents of ready-to-eat chocolate cereals. Journal of the American Dietetic Association, v. 100, n. 6, p. 690-692, 2000.

CHEN, Q. et al. Simultaneous determination of caffeine, theobromine and theophylline in foods and pharmaceutical preparations by using ion chromatography. Analytica Chimica Acta, v. 371, n. 2-3, p. 287-296, 1998.

DUTCOSKY, S. D. Aditivos associados às características sensoriais. Disponível em:<http://www.pucpr.br/educacao/academico/ graduacao/cursos/ealimentos/ppt/aditivosensorial.ppt $>$. Acesso em: 30 Jan. 2006.

EDUARDO, M. F. Avaliação reológica e físico-química de achocolatados e bebidas achocolatadas. São Paulo, 2005. 108p. Dissertação (Mestrado em Ciências Farmacêuticas) - Faculdade de Ciências Farmacêuticas, Universidade de São Paulo.

ETENG, M. U. et al. Recent advances in caffeine and theobromine toxicities: a review. Plant Foods for Human Nutrition, v. 51, n. 3 , p. 231-243, 1997.

FERREIRA, V. L. P. et al. Análise sensorial: testes discriminativos e afetivos. Campinas: SBCTA, 2000. P. 1-6. (Manual: Série Qualidade).

GRAB, W.; BRUGGER, W.; TAYLOR, D. Cocoa substitution. In: CHARALAMBOUS, G.; INGLETT, G. E. Flavor of Foods and Beverages: Chemistry and Technology. New York: Adacemic Press, 1978. p. 81-90.

GROSSO, L. M.; BRACKEN, M. B. Caffeine metabolism, genetics, and perinatal outcomes: a review of exposure assessment considerations during pregnancy. AEP, v. 15, n. 6, p. 460-466, 2005.
INSTITUTO ADOLFO LUTZ. Normas analíticas do Instituto Adolfo Lutz. 2 ed. São Paulo, 1976. v. 1, p. 14-63

KUMAZAWA, S. et al. Antioxidant activity of polyphenols in carob pods. Journal of Agricultural and Food Chemistry, v. 50, n. 2, p. 373-377, 2002.

LANNES, S. C. S.; MEDEIROS, M. L. Processamento de achocolatado de cupuaçu por spray-dryer. Revista Brasileira de Ciências Farmacêuticas, v. 39, n. 1, p. 115-123, 2003.

MATISSEK, R. Evaluation of xanthine derivates in chocolate: nutricional and chemical aspects. Zeitschrift für Lebensmittel-Untersunghung und Forschung, v. 205, n. 3, p. 175-184, 1997.

MEDEIROS, M. L. Estudo a aplicação de substitutos de cacau. São Paulo, 2006. Dissertação (Mestrado) - Faculdade de Ciências Farmacêuticas, Universidade de São Paulo.

MEDEIROS, M. L.; LANNES, S. C. S. Torta de cupuaçu: composição e utilização. Revista Brasileira de Ciências Farmacêuticas, v. 35, supl. 1, p. 118, 1999.

NAZARÉ, R. F. R. Produtos agroindustriais de bacuri, cupuaçu, graviola e açaí, desenvolvidos pela Embrapa Amazônia Oriental. Belém: Embrapa Amazônia Oriental, 2000. 27 p.

OMOBUWAJO, T. O.; BUSARI, O. T.; OSEMWEGIE, A. A. Thermal agglomeration of chocolate drink powder. Journal of Food Engineering, v. 46, n. 2, p. 73-81, 2000.

OWEN, R. W. et al. Isolation and structure elucidation of the major individual polyphenols in carob fibre. Food and Chemical Toxicology, v. 41, n. 12, p. 1727-1738, 2003.

QUEIROZ, M. B.; GARCIA, N. H. P. Avaliação da torração de amêndoas de cupuaçu (Theobroma grandiflorum). Brazilian Journal of Food Technology, v. 2, n. 1,2, p. 167-173, 1999.

REISDORFF, C. et al. Comparative study on the proteolytic activities and storage globulins in seeds of Theobroma grandiflorum (Willd ex Spreng) Schum and Theobroma bicolor Humb Bonpl, in relation to their potencial to generate chocolate-like aroma. Journal of the Science of Food and Agriculture, v. 84, n. 7, p. 693-700, 2004.

THOMAS, J. B. et al. Determination of caffeine, theobromine, and theophylline in standard reference material 2384, baking chocolate, using reverse-phase liquid chromatography. Journal of Agriculture and Food Chemistry, v. 52, n. 11, p. 3259-3263, 2004.

VERPOORTE, R. Methods for the structure elucidation of alkaloids. Journal of Natural Products, v. 49, n. 1, p. 1-25, 1986.

YANG, H. et al. New bioactive polyphenols from Theobroma grandiflorum ("Cupuaçu”). Journal of Natural Products, v. 66, n. 11, p. 1501-1504, 2003.

YOUSIF, A. K.; ALGHZAWI, H. M. Processing and characterization of carob powder. Food Chemistry, v. 69, n. 3, p. 283-287, 2000. 